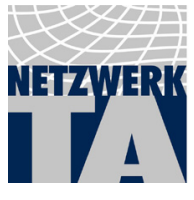

\section{Jahrestreffen 2011}

Das Jahrestreffen des Netzwerks Technikfolgenabschätzung (NTA) fand am 21. November 2011 im Zentrum Technik und Gesellschaft (ZTG) der TU Berlin statt. Das Koordinationsteam berichtete über die Aktivitäten des vergangenen Jahres und die Planung der NTA5 (siehe Ankündigung unten). Die Arbeitsgruppe „IuK“ berichtete unter anderem von einem DFG-Antrag für ein TAFachportal (der Ende Januar 2012 genehmigt wurde). Das Netzwerktreffen wurde abgerundet mit der Vorstellung des EU-Projekts PACITA (Parliaments and Civil Society in Technology Assessment), an dem einige institutionelle Mitglieder des NTA beteiligt sind und das darüber hinaus für die „TA-Gemeinde“ interessant ist, da es die stärkere Verbreitung insbesondere parlamentarischer TA-Ansätze in Europa zum Ziel hat.

\section{Workshop der AG „Governance“}

Der Workshop „Theorie und Praxis von Technology Governance. Fragestellungen und Erkenntnisse aktueller Forschung im Kontext Technology Assessment und Governance“", organisiert von der Arbeitsgruppe „TA und Governance“ des NTA und namentlich von Stephan Bröchler, Petra Schaper-Rinkel und Claus Seibt, fand im Anschluss an das Jahrestreffen ebenfalls im ZTG statt. Ein Bericht zu dem Workshop findet sich in dieser Ausgabe von TATuP (Rubrik Tagungsberichte).

\section{TRANSDISS verlängert}

Das Doktorandenprojekt „Disziplinäre Forschung in der Transdisziplinarität. Dissertationen in der Technikfolgenabschätzung" (TRANSDISS) wurde seitens des Bundesministeriums für Bildung und Forschung verlängert. Doktorandinnen und Doktoranden, die sich im Bereich der Technikfolgenabschätzung mit transdisziplinä- ren Fragestellungen befassen, sind herzlich eingeladen, sich zu bewerben.

\section{NTA5 - Die fünfte Konferenz des Netzwerks TA findet in der Schweiz statt}

„Vordenken - mitdenken - nachdenken. Technikfolgenabschätzung im Dienst einer pluralistischen Politik" ist der Titel der 5. Tagung des NTA, die vom 29. bis 31. Oktober 2012 in Bern stattfinden wird. Integriert in das Tagungsprogramm ist auch die Jubiläumsfeier 20 Jahre TASWISS. Der Call for Abstracts endet am 29. Mai 2012. Der Text des Calls beginnt mit einem Zitat: „Was einmal gedacht wurde, kann nicht mehr rückgängig gemacht werden“, schreibt Friedrich Dürrenmatt in „Die Physiker" und weiter: „Was alle angeht, können nur alle lösen“". Gesellschaftlicher Kernauftrag der politikberatenden Technikfolgenabschätzung ist es, intendierte und nicht intendierte Folgen, Chancen und Risiken neuer Technologien bzw. technischer Neuerungen frühzeitig zu erkennen und zu untersuchen.

Diese TA betreibt ihre Aufgabe nicht als Selbstzweck: Die (unvoreingenommenen und wissensbasierten) Ergebnisse ihrer Auseinandersetzungen sollen politischen Entscheidungstragenden als Grundlage dienen, um technologiepolitische Weichen so zu stellen, dass die Gesellschaft daraus den größtmöglichen Gewinn zieht und den kleinstmöglichen Schaden erleidet. Die TA zeigt Fakten auf, entwickelt Leitbilder und normative Konzepte mit, untersucht Nutzungs- und Wirkungskontexte von neuen Technologien, unterstützt innovationsund lösungsorientierte Strategien, orientiert Entscheidungstragende und die Öffentlichkeit, liefert Argumente, formuliert Empfehlungen, versachlicht Debatten und Kontroversen. Die Verantwortung für die Konfliktlösung liegt auf politischer und gesellschaftlicher Ebene.

Sollte Ihr Interesse geweckt sein, finden sie hier den Link: http://www.netzwerk-ta.net/termine.htm\#desNTA. 